\section{Rumen metagenomics}

The cost-effectiveness of biofuel may depend on the availability of better lignocellulolytic enzymes. An ideal source of these catalysts would be microbes in cow rumen, which digest complex plant polysaccharides with remarkable efficiency. Yet most members of the rumen microbiome defy culture.

Hess et al. incubated nylon bags containing switchgrass, a promising energy crop, in the stomachs of fistulated cows for $72 \mathrm{~h}$. Organisms adhering to the partially digested plant material were sequenced, yielding $268 \mathrm{Gbp}$ of data and $>2.5$ million coding sequences. The authors identified $>27,000$ genes encoding putative carbohydrate-active enzymes, $43 \%$ of which are novel (having $<50 \%$ identity to known proteins). Of 90 candidate gene products tested for activity, 51 were active on at least one of ten different substrates, including two potential biofuel feedstocks. Further analysis of this catalog of biomassdegrading enzymes may help to overcome a major bottleneck in biofuel production. (Science 331, 463-467, 2011)

\section{Single-molecule imaging}

Pulses of extremely bright X-rays have been proposed as tools for working out the structures of large biological macromolecules that cannot be determined by other methods. Seibert et al. report on progress toward this goal. They show diffraction patterns that result from pulses of $1,700,000$ photons being scattered by single capsids of the $0.45 \mu \mathrm{m}$ mimivirus, which is too large for cryoelectron microscopy and cannot be crystallized owing to a dense outer layer of fibrils attached to the capsid. The diffraction patterns can be reconstructed into an image having an estimated resolution of $32 \mathrm{~nm}$. In principle, this approach can achieve a resolution of $1 \mathrm{~nm}$ or less, but the linear particle accelerator used in these experiments was not powerful enough to attain this limit. Notably, the method does not require modifications to the sample, such as staining, freezing, sectioning, radiolabelling or crystallization. (Nature 470, 78-81, 2011) CM

\section{Chromatin determines transcription factor binding}

For most transcription factors, only a fraction of potential binding sites is occupied by the protein at any given time. What accounts for this selectivity is largely unknown. John et al. demonstrate in genome-wide experiments that the binding behavior of a glucocorticoid receptor is determined largely by chromatin structure. They investigate the relationship between the binding sites of the receptor after hormone stimulation with the pre-existing patterns of chro-

Written by Kathy Aschheim, Laura DeFrancesco, Markus Elsner,

Peter Hare \& Craig Mak matin accessibility in two cell types. Their comparison of ChIP-seq data with analysis of DNA accessibility by digital DNase I profiling reveals that up to $95 \%$ of the binding occurred at sites with an open chromatin formation. The dominant effect of chromatin structure can be modulated by the specific glucocorticoid receptor binding element present, as some motifs are more likely to be bound even if the DNA is inaccessible to DNase I, and by nearby binding sites of additional co-regulatory factors, such as AP-1. As glucocorticoids are widely used to combat inflammatory, autoimmune and allergic conditions, the study raises the intriguing possibility that patient responses to nuclear receptor ligand drugs might be stratified on the basis of epigenetic status (Nat. Genet. published online, doi:10.1038/ ng.759, 23 January 2011)

$M E$

\section{Profiling cancer drugs}

Chemogenetic profiling screens a drug against a panel of genetic mutants to predict the drug's mechanism of action. This strategy has been demonstrated using yeast deletion mutants (deleting a drug target creates drug resistance), and something analogous has been attempted with panels of cancer cell lines. However, not all mammalian pathways are represented in yeast, and cancer cell lines have drawbacks that limit their broad application. Jiang et al. describe a chemogenetic profiling approach feasible in mammalian cells that uses RNA interference to knock down genes in pathways putatively targeted by drugs. In a proof-of-principle experiment, they tested 15 chemotherapy drugs in a Burkitt's lymphoma cell line, chosen for its sensitivity to a broad range of drugs. Lines transfected with individual short hairpin RNAs (shRNAs) directed against 29 genes central to cell-fate decisions were treated with the drugs, creating a pattern of resistance for each drug across the set of cultures. Drugs with similar modes of action are expected to have similar patterns of resistance, and in fact the 15 drugs tested fell into six clusters, according to their modes of action. With this system, the authors tested three approved drugs with unknown mechanisms, which led to a seventh cluster, and characterized a derivative of a nitrogen mustard, finding that its mode of action was unchanged, even though the toxicity was increased. Finally, they showed that profiling an additional 16 known drugs with a subset of 8 of the initial 29 shRNAs was sufficient to correctly predict the drugs' mechanisms of action. (Nat. Chem. Biol. 7, 92-100, 2011)

$L D$

\section{Off-the-shelf blood vessels}

Tissue-engineered blood vessels would be useful for certain cases of arterial disease that are not amenable to autologous or synthetic grafts. Blood vessels have been generated in vitro for autologous transplants by generating sheets of tissue from fibrolasts, wrapping the sheets around rods to form tubes, and seeding the lumens with endothelial cells. But this patient-specific method is costly and requires 6-9 months of culture time. Dahl et al. propose a more practical solution based on allogeneic cells. They seed smooth muscle cells on a biodegradable polymer scaffold and allow the cells to secrete extracellular matrix proteins, such as collagen. Then the construct is decellularized to remove immunogenic material, leaving a collagenous tube suitable for transplantation. The authors test the approach in animal models of arteriovenous access for hemodialysis and of peripheral and coronary artery bypass. The grafts remain patent for up to 6 months and are resistant to dilatation, calcification and intimal hyperplasia. (Science Transl. Med. published online, doi: 10.1126/scitranslmed.3001426, 2 February 2011)

$K A$ 